DOI: 10.4312/an.50.1-2.189-201

\title{
Beatrix, un roman courtois au sein du cycle de la croisade
}

\section{Tadeja Dermastja}

\section{Synopsis}

Dans cet article on essaie d'exposer certains éléments du fabuleux dans la branche médiévale de Beatrix qui a été intégrée au cycle épique français de la croisade et la comparer au poème médiéval anglais portant le même titre. La question qui se pose dans ce sens est pourquoi le thème de cette chanson pouvait-il être intéressant dans les deux milieux littéraires et quelles sont les circonstances historiques et littéraires qui l'ont fait connu dans les différents cadres culturels européens. Il faudra aussi rechercher tous contextes de cette matière qui est entrée dans le genre épique, mais qui pourrait représenter un autre genre poétique médiéval; que ce soit la poésie courtoise soit la poésie chevaleresque, la branche de Beatrix a été jointe aux chansons des croisades, celles-ci étant majoritairement les chansons de geste. La branche de Beatrix raconte l'histoire de la naissance du Chevalier au cygne, dont la légende était très répandue dans le continent européen, surtout sous la forme de la légende de Lohengrin. On démontrera que la matière de Beatrix était connue en Angleterre pour des raisons purement historiques et qu'en effet cette matière y a préexisté et elle lui a été rendue après sêtre proliférée dans le continent.

Mots-clefs: Chanson de geste, Godefroi de Bouillon, Cycle de la croisade, branche de Beatrix, croisades, poésie médiévale, fabuleux 
La chanson de geste s'appuie autant sur les chroniques que sur les légendes, elle s'éloigne pourtant des épopées homériques, demeurant à l'écart des mythologies où il y a les mélanges du monde des dieux et des hommes et s'approchant des sagas qui racontent les histoires des familles où le monde des dieux disparaît. La matière épique française est fortement basée sur l'époque carolingienne, les hagiographies, les traditions orales, les chroniques latines et sur l'idéologie féodale, ce qui crée une mythologie particulière, car elle implique l'imaginaire chrétien et, très rarement, le monde ontologique provenant des légendes antérieures à la christianisation de l'Ouest. La pléiade des chansons de geste réunies aux cycles, fait partie de la matière épique du Charlemagne, ce qui représente la partie majeure des chansons de geste en France.

En dehors de la matière carolingienne qui est souvent reprise en trois cycles (Labarthe, 2006 : 83) se trouvent les épopées des croisades qui n’ont été étudiées de près que récemment et les chercheurs contemporains en distinguent amplement deux cycles (Labarthe, 2006 : 99 ; cf. Bender, 35-83) : le premier cycle comprendrait la Chanson d'Antioche en tant que chanson principale du cycle et le deuxième, les chansons ayant pour noyau la chute de Jérusalem. Pourtant, ces classifications sont faites du point du vue historique et non-contextuel, parce que presque toutes les chansons de ce cycle relatent les épisodes de la vie du héros Godefroi de Bouillon, c'est pourquoi les classifications récentes redoublent la présence de certaines chansons dans les deux cycles (p.ex. les branches relatant l'enfance du héros sont dans les deux cycles) et Judith Labarthe constate que: "Les deux cycles forment un sous-genre épique non homogène. Ils sont constitués d'une série de formes limites, se combinant chaque fois avec des genres apparentés comme l'historiographie, le roman chevaleresque, le conte; la latitude dont jouit le genre épique s'y manifeste pleinement » (Labarthe, 2006 : 99 - 100).

Godefroi de Bouillon, chef militaire de la première croisade, représente l'actant principal de plusieurs branches de nature biographique auxquelles sont rajoutées les branches intitulés Beatrix et Elioxe (les deux représentent $\mathrm{La}$ Naissance du Chevalier au Cygne $)^{1}$. Aussi celles qui puisent des éléments biographiques ne peuvent pas représenter la chanson de geste au sens propre, elles sont un mélange des genres, allant du conte au roman chevaleresque, transfigurant la chanson de geste en fabuleux des légendes du monde ontologique. Ce qui

1 Dans l'édition intégrale de tous les manuscrits du Cycle de la croisade, plusieurs auteurs américains ont donné les noms suivants aux manuscrits contenant les poèmes versifiés, publiées en huit volumes dans la publication de l'Université d'Alabama : La Naissance du Chevalier au Cygne (avec les versions d'Elioxe et de Beatrix), Le Chevalier au Cygne, La fin d'Elias, Les enfances Godefroi, La Chanson d'Antioche, Les Chétifs, La Chanson de Jérusalem; s'ensuivent les « continuations » de Jérusalem : La Chrétienté Corbaran, La Prise d'Acre, La Mort Godefroi et La Chanson des Rois Baudouin. (Mickel et Nelson, 1977 : xi). 
serait intéressant d'analyser de plus près est ce fabuleux qui est entré dans les littératures française, anglaise, espagnole et allemande, cette dernière inaugurant l'histoire du chevalier au cygne dans la saga de Lohengrin, interprété aussi en musique wagnérienne. Bien que la légende de Lohengrin soit bien analysée dans le milieu allemand, il y a toujours quelques lacunes dans les études médiévales anglaises et françaises à propos de cette matière. La question qui se pose est pourquoi a-t-on fait si peu de recherches du fabuleux des épopées des croisades, même si celui-ci était très répandu à l'époque et assez populaire pour s'étaler en Europe occidentale et en Angleterre ?

L’herméneutique littéraire et les études de l'érudition du XIX ${ }^{\mathrm{e}}$ siècle laissaient de côté, dans une certaine mesure, les analyses plus approfondies de cette matière à cause des désagréments politiques trop nationalistes. Pourtant, Judith Labarthe reprend la constatation de David Jacoby que même au Moyen-Âge «les croisés, [...] apprécient d'ailleurs davantage les chansons d'outre-mer» (Labarthe, 2006 : 98) ce qui d'ailleurs confirme la popularité du cycle carolingien, mais qui prouve qu'à l'époque, l'armée croisée autant que la population qui l'accompagnait connaissait une pléiade de genres épiques. Dans les analyses des épopées, les recherches doivent nécessairement porter dans la même mesure aux faits historiques et à la science littéraire et c'est dans ces deux directions qu'il faut analyser le fabuleux du Chevalier au Cygne et surtout les faits qui répandaient sa popularité en France et en Angleterre et qui présentent une lacune d'analyses dans les sciences littéraires d'aujourd'hui. Ce fabuleux qui sera le sujet du présent article, n'est pas en majorité la légende du chevalier mystérieux qui se transfère dans un petit bateau mené par son frère en forme du cygne, mais le conte de sa mère, qui prend soit une forme divine soit une forme humaine et dont la branche fait partie initiale du Cycle de la Croisade qui commence par l'histoire biographique de Godefroi de Bouillon.

La matière pour ce conte qui comprend tous les éléments d'un conte de fée selon la Morphologie du conte de Vladimir Propp ${ }^{2}$ n'existe pas en version allemande du Lohengrin où le chevalier au cygne est le fils de Parzival allemand, pourtant on trouve le même conte ayant les noms différents en Angleterre, en Espagne et surtout en France (Lecouteux, 1998 : 105). Le sujet de l'analyse dans cet article porte sur une étude comparative de ce conte et de ses sources dans les littératures anglaise et française et sur l'actualisation de ces légendes fabuleuses médiévales.

Pour dévoiler le phénomène de leur présence sur le continent européen et en Angleterre, il faut présenter quelques points historiques et sommairement toucher au contenu du conte. Le manuscrit portant le titre latin Dolophatos, sive de Rege et Septem Sapientibus datant du XII e siècle a été écrit par le moine lorrain Jean de

2 Surtout ses recherches des transformations des contes merveilleux. Voir Propp, La Morphologie du conte. Paris : Seuil, 1970, p. 172-249. 
Haute-Seille où on trouve sept contes indoeuropéens, le conte en question étant présenté le dernier, le septième (Nelson et Mickel 1977 : 1xxxxii). Tout à la fin de son livre, l'auteur mentionne qu'il a utilisé le matériel de la tradition orale, ce qui peut, selon Huet, prouver que l'auteur a entendu ce conte d'un jongleur qui a déjà chanté des exploits de Godefroi de Bouillon incluant, parmi les chansons de la croisade, ce conte fabuleux de sa naissance (Ibid., cxiv).

Le récit du conte qui fait partie des chansons des croisades se résume ainsi : Beatrix et le roi Oriant sont un couple sans enfants et de leur donjon ils observent une femme avec les enfants jumeaux. Beatrix médit de cette femme en disant que les enfants jumeaux doivent certainement être produits de l'adultère. Cette nuit même elle devient enceinte et par la suite elle accouche de sept enfants qui sont nés avec les chaînes en argent autour du cou. La mère du roi, Matabrune, est une femme d'une méchanceté extraordinaire et elle ordonne à son servant Macron de porter les enfants dans la forêt et les tuer. Celui-ci ne les tue pas, il les expose dans la forêt où il y a l'ermite qui sauve les enfants. Matabrune explique à son fils que pendant son absence, Beatrix a accouché de sept chiens et qu'elle doit être tuée. Le roi Oriant jette Beatrix à la prison. Après des années, un autre servant de Matabrune, Malquarés, trouve les enfants dans la forêt et immédiatement il reçoit l'ordre de la vieille reine d'apporter les chaînes. Il réussit, mais le garçon favori de l'ermite était dans la forêt avec lui. Les six enfants changent aux cygnes et s'envolent au marais de leur père. Mataburne donne les chaînes à l'orfèvre pour en faire les coupes, mais celui-ci trouve que les chaînes sont magiques et produit deux coupes d'une seule chaîne. Il garde les autres chaînes. Après quinze ans, Matabrune convainc son fils de brûler Beatrix. Elle pourrait être sauvée au cas où elle trouve un champion pour la défendre. En ce moment un ange apparaît au garçon et à l'ermite qui leur explique la situation et envoie le garçon défendre sa mère. Celui-ci était élevé comme Perceval et il ne connaît rien des combats chevaleresques, mais il est instruit que Dieu le protégerait. Dès son arrivée dans la ville, il est baptisé au nom d'Elias et il gagne le combat, libère sa mère et garantit la transformation des enfants-cygnes qui reprennent la forme humaine. Seul un frère reste dans sa forme animale. Matabrune s'enfuit dans son château et Elias vient l'assiéger, il gagne de nouveau et Matabrune est brûlée au bûcher préparé pour Beatrix. En respectant la volonté d'un ange, Elias et son frère-cygne s'en vont par la mer et après encore une bataille victorieuse, ils arrivent à Nimègue. ${ }^{3}$

La version du conte de Beatrix diffère de celle de Dolopathos et d'Elioxe dans plusieurs éléments et elle semble être adaptée pour pouvoir fonctionner dans l'épopée de la croisade et surtout dans les chansons qui touchent à la poésie de

3 Le texte est résumé d'après la lecture des manuscrits publiés en édition de "The Old French Crusade Cycle» (Nelson et Mickel, 1977 : 131 - 201). 
Godefroi de Bouillon. Les manuscrits en français pour la version de Beatrix se trouvent dans la Bibliothèque nationale ${ }^{4}$, dans la Bibliothèque d'Arsenal à $\mathrm{Pa}$ ris $^{5}$ et à British Museum à Londres ${ }^{6}$. Pourtant, il existe la branche de Beatrix en anglais, intitulé Chevalere Assigne et se trouve aussi à British Museum dans un seul manuscrit ${ }^{7}$ dans une version très abrégée, n'ayant que 370 lignes et menant l'histoire jusqu'à la reprise de la forme humaine des enfants-cygnes. Le manuscrit porte la date de la deuxième moitié du XIV e siècle et il est écrit dans l'accent de l'anglais moyen ${ }^{8}$ dans les vers allitérés. L'édition intégrale et annoté a été publiée en 1868 par Henry Gibbs. Le copiste du manuscrit anglais dit précisément qu'il puise la matière d'un autre livre :

«The kynge hette oryens - as Pe book tellethe » (v. 7)

«They kallede hym Enyas to name - as Pe book tellthe » (v. 270) (Gibbs, 1868 $: 1,14)^{9}$

Il est possible que le copiste ait utilisé des sources littéraires pour remanier la matière qui était très répandue en Europe, mais qui n'existait pas en langue anglaise. Cependant, c'est justement cette matière fabuleuse qui est venue d'Angleterre et elle lui a été rendue, après être imprégnée dans les chansons de geste. Les raisons pour lesquelles elle est retournée en Angleterre résident dans le fait que les ancêtres et les descendants de Godefroi de Bouillon étaient liés à l'histoire d'Angleterre.

Avant la prise de la ville de Jérusalem en 1099 par les croisés et son titre du « roi de Jérusalem ", Godefroi était le comte de Boulogne. Le père Eustache II, était parmi les soldats de Guillaume le Conquérant et à cause de ses engagements guerriers, il a reçu les propriétés en Angleterre, que sa mère Ide, béatifiée après sa mort,

4 Les manuscrits des fonds français aux indices 786, 795, 1621, 12569 (Nelson et Mickel, 1977 : xxiv $-1 \mathrm{xv})$

5 Portant l'indice de 3139 (Nelson et Mickel, 1977 : xlv).

6 Portant les indices 3661 et 15 E VI.

7 Portant l'indice Cotton Caligula A. II (Barron, $1967: 25)$.

8 Laccent dans le poème est soumis aux analyses. J.R.Hulbert qui cite R. Morris constate que « the Dialect [of Chevalere Assigne] in its present form is East Midland. But as we do not fint [other] East Midland writers adopting alliterative measures in the 14th century, I am inclined to think that the original English text was written in the N. or N.W. of England. » (Hulbert, 1921 : 5). Pourtant, C. Moorman qui recherche le renouveau de la poésie allitérative du XIV siècle en Angleterre constate que «twelve alliterative poems written between 1350 and 1400 in the Western and Notrhern Midlands [...] » et parmi ces douze poèmes en allitération il cite aussi la chanson de Chevalere Assigne (parmi Winner and Waster, Morte Artur, Sir Gawain and the Green Knight etc.)

9 Le livre concerné peut être le manuscrit français $15 \mathrm{E}$ VI à Londres, mais ce n'est pas évident (Barron, $1967: 32$ ). 
gérait bien après être devenue veuve. ${ }^{10}$ Ide de Boulogne a vendu quelques-unes de ses propriétés en Angleterre pour financer l'exploit de la croisade de ses trois fils, mais après celles-ci son fils aîné a eu la fille Mathilde qui est devenue la reine d'Angleterre après son mariage à Étienne de Blois ${ }^{11}$. La présence de la famille de Boulogne sur le territoire anglais est évidente aussi dans l'une des branches du Cycle de la croisade, celle de la chanson des Enfances Godefroi où on trouve le frère de Godefroi, Eustache à Londres pour son éducation chevaleresque.

"Que Witasses l'aisnés ot .xii. ans acomplis,

Moult estoit prox et sages, cortois et bien pris;

N'avoit si bel vaslet el regne Loéis.

Par le los que li quens ot de ses bons amis,

L'a al roi d'Engleterre por adober tramis. » (v. 761-765)

Le roi cité dans la chanson est un des rois capétiens, mais celui-ci n'est pas historiquement contemporain à Godefroi de Bouillon (Mickel 1999 : 119). La grandeur historique de Godefroi réside dans le fait qu'il était le roi de Jérusalem. Son règne était très court et après sa mort son frère Baudouin a accédé au trône. Un des chroniqueurs, Guillaume de Tyr, constate avec indignation dans la seconde moitié du XII ${ }^{\mathrm{e}}$ siècle : "Jomets avec intention la fable du cygne, rapportée cependant dans un grand nombre de récits, et qui a fait dire vulgairement que les fils du comte Eustache avaient eu une naissance merveilleuse : mais une telle assertion parait trop contraire à toute vérité » (Guizot, $1824: 11){ }^{13}$

De cela s'ensuit que les jongleurs ont, déjà à cette époque, relié l'histoire biographique aux récits légendaires. ${ }^{14} \mathrm{Du}$ point du vue historique, la vie de Godefroi de Bouillon dévoile que c'était un homme très militaire, mais à la fois humble ; Guillaume de Tyr note qu'il a résigné à porter la couronne de la ville sainte en disant

10 Le comte Eustache II. de Boulogne a reçu douze fiefs dans le Hertford, l'Essex, le Norfolk, le Bedford et le Kent. Sur ce point, après les exploits militaires avec Guillaume le Conquérant, le comté de Boulogne a sa plus grande étendue (Dickès 2004 : 61).

11 Ibid., 216.

12 Les vers sont tirés de l'édition du XIX ${ }^{\mathrm{e}}$ siècle, faite par Célestin Hippeau qui était le seul à publier quelques manuscrits du fonds français, notamment celui portant l'indice 1621 qui fait partie du fonds français, mais sa publication porte le titre La Chanson du Chevalier au Cygne et de Godefroi de Bouillon (Hippeau, 1969 : 28).

13 Le texte en latin en original : "Praeterimus denique studiose, licet id verum fuisse plurimorum astruat narratio, Cygni fabulam, unde vulgo dicitur sementiam eis fuisse originem; eo quod a vero videatur deficere alis assertio.» Http://www.thelatinlibrary.com/williamtyre/9.html, consulté le 26 juin 2017.

14 Il y a une lettre de Guy de Bazoches vers 1175 constatant l'ascendance féerique de la famille de Bouillon, mais Guillaume de Tyr la rejette comme fabuleuse. (Gaullier - Bougassas, 2005 : 116) 
que "son extrême humilité le porta à ne point vouloir être distingué dans la Cité sainte par une couronne d'or semblable à celle que portent les rois» (Guizot, 1824 : 17). Même si Godefroi est mort après avoir régné une seule année, c'est son frère Baudouin qui a continué la régence à la ville sainte. Ce qu'il faut rechercher davantage c'est aussi la corrélation de la famille de Boulogne - Bouillon à l'établissement de l'ordre templier. Lordre s'est développé au fur et à mesure de la « garde du Saint-Sépulcre » qui protégeait la communauté des chanoines du Saint-Sépulcre établie par Godefroi de Bouillon (Demurger, 2008 : 25). Plus tard, la reine Mathilde établissait des églises templières en Angleterre et supportait l'ordre des templiers. ${ }^{15}$

En tant que héros littéraire, Godefroi occupe le rôle central dans presque toutes les chansons de la croisade, sauf dans les deux chansons fabuleuses de sa naissance qui ont été probablement jointes à posteriori dans le récit biographique de son enfance et dans $L a$ Chanson d'Antioche qui met en valeur d'autres héros importants pour leurs exploits militaires. Cette chanson est d'ailleurs la branche centrale du Cycle de la croisade et elle s'est probablement développée pendant la croisade ellemême. C'est la gloire de Godefroi de Bouillon qui a rendu populaires d'autres chansons, entre autres les récits légendaires de Beatrix et d'Elioxe.

Le rôle de Godefroi en tant que héros littéraire semble être celui de Charlemagne, mais dans une mesure beaucoup plus restreinte. On y trouve aussi des éléments d'autres œuvres littéraires médiévales, notamment de La Chanson de Roland. Les trouvères et les jongleurs y ont inséré le détail avec Perceval, ce qui montre qu'ils connaissaient le roman courtois. Ide de Boulogne joue aussi un rôle majeur dans le Les Enfances Godefroi, car étant la mère de Godefroi qui portait la couronne de Jérusalem elle pourrait prendre un semi - rôle de la sainte Marie, c'est pourquoi elle vit dans la littérature les moments qui ressemblent à ceux de Beatrix. Dans le Cycle de la croisade, elle est la petite-fille de Beatrix et la fille du Chevalier au cygne et avant la naissance de ses trois fils, elle est confrontée aux visions pareilles à celles de sa grand-mère, mais à ce point, le fabuleux qui semblait provenir d'un monde païen, prend l'aspect chrétien.

Dans le genre épique, les héros font souvent partie de l'au-delà (p.ex. Achille, fils d'un mortel et d'une nymphe) ou sont au moins à la recherche de l'immortalité (p.ex. Gilgamesh). Contrairement aux héros grecs antiques qui sont d'après Daniel Madelénat «des dieux déchus, ou des hommes promus " (Madelénat, 1986 : 54), le héros médiéval et celui des chansons de geste françaises restent dans le cadre du réel, parce qu'il est souvent un roi, un chevalier, un noble, mais pareillement aux grands épopées antiques, ce héros provient parfois du monde ontologique, en possédant, comme le résume Charles Baudoin un signe de reconnaissance, qui se

15 À comparer : Chibnall, Marjorie, "Empress Matilda : Queen Consort, Queen Mother and Lady of the English » London : Blackwell Publishers, 1993. 
manifeste soit par l'origine royale du héros soit par son enfance mystérieuse et ses actes extraordinaires (Baudoin, 1952).

Pour le héros médiéval, il est nécessaire de garder un certain mystère chrétien, impénétrable pour le public et qui crée une certaine distance car le Dieu chrétien ne peut pas coexister avec des hommes, mais dans les hommes. Daniel Madelénat en citant Hegel constate que le merveilleux doit "préserver l'indépendance [...] des actions humaines et des actions divines, afin que les dieux n'apparaissent pas comme des abstractions sans vie et que les individus humains ne soient pas dégradés au niveau de simples serviteurs. » (Madelénat, 1986 : 63). De cela s'ensuit que le seul héros qui puisse être capable à représenter un idéal chevaleresque dans le cas des chansons de geste, serait celui qui joindrait l'image de l'homme et l'hypostase du Dieu et Godefroi en est l'exemple par excellence. Même dans les drames modernes il y a toujours cette présence du surnaturel, c'est notamment Paul Claudel qui dit à propos de son drame aux dimensions épiques, Soulier de satin, que ses personnages surnaturels entrent dans sa composition, parce que leur présence lui a paru artistiquement nécessaire, "de même que dans l'Iliade l'absence des personnages de l'Olympe représenterait quelque chose d'absolument inconcevable »(Claudel, 1954 : 276). Le héros épique est souvent l'hypostase d'une nation, et souvent il est "dépourvu d'biatus entre un moi et une persona, [...] il porte à leur plus haut degré les qualités requises par l'action [...]» (Madelénat, 1986 : 55) et c'est pour cette raison que Roland peut être à la fois courageux comme il sauve l'armée de Charlemagne, mais il a peur de sonner le cor, pour qu'on ne fasse pas de lui une " male chanson ». ${ }^{16}$ C'est pareil pour le chevalier au cygne, qui subit à l'interdiction mystérieuse : il quitte son pays avec son frère cygne après la question interdite de « la dame du Bouillon ».

L'histoire fabuleuse prend fin avec le départ d'Elias et sur ce point commencent les exploits guerriers, le thème central des épopées et des chansons de geste. L'épopée est aussi le mythe de la guerre, son herméneutique, et l'historiographie, pareille à celle des chroniques. Le conflit qui est l'immanence humaine y est mis au piédestal comme la vérité singulière de l'homme et la guerre qui, d'après la philosophie allemande de Marx et Hegel, repose " au cour de la pensée et de la société, et en la justifiant comme voie vers la fin de l'bistoire» (Ibid., 68). L'épopée trouve un point de lumière dans la sombre atmosphère de la guerre et c'est l'amour pour la gloire du héros, un amour sacré pour la reconnaissance parmi les siens.

Le but des chansons de geste était souvent le divertissement de la population noble à la cour, surtout lors des adoubements, les tournois, les banquets où il $\mathrm{y}$ avait les présentations des armes, des chevaux (Labarthe, 2006 : 78). C'étaient des occasions où tout le monde de la cour était présent et pour cela il est tout à fait vraisemblable que pendant les événements de société il y avait aussi les dames de 
la cour. Même si l'enchaînement des branches du Cycle de la croisade était faite à posteriori et il y a toujours quelques ambigüités dans leur disposition dans le cycle $^{17}$, on peut définitivement constater que les deux récits de La Naissance du Chevalier au cygne étaient intégrés à l'histoire du héros parce qu'il nous reste deux manuscrits en prose, l'un datant du XIV ${ }^{e}$, l'autre du XV siècle, ce dernier étant d'un grand intérêt pour Marie de Clèves qui se croyait la descendante de la famille Boulogne-Bouillon et qui intègre encore une petite partie des Enfances de Godefroi (Emplaincourt, 1989 : xiii - xxx). ${ }^{18}$

La version anglaise ne possède que ce fabuleux qui pourrait présenter dans le cas de deux versions françaises, soit Elioxe, soit Beatrix le roman courtois indépendant. Où peut-on trouver la source de la légende qui ouvre l'histoire biographique de Godefroi et des croisades ? On vient de prouver que le récit légendaire de la famille Boulogne - Bouillon pourrait intéresser le monde des lettres anglaises, mais il n'est quand même pas évident, si la légende pourrait intéresser un public plus large. Cathérine Gaullier - Bougassas en citant Georges Duby souligne qu'il $\mathrm{y}$ avait pratique au $\mathrm{XII}^{\mathrm{e}}$ et au $\mathrm{XIII}^{\mathrm{e}}$ siècles dans les familles aristocratiques de se trouver des ancêtres ayants une source généalogique féerique (Gaullier - Bougassas, 2005 : 116) et elle cite encore les romans lignagers en anglo-normand qui, de cette façon célèbrent une famille aristocratique. S’il y a, dans ce territoire, la tradition de ce genre, il n'est pourtant pas extraordinaire de trouver la légende des enfants-cygnes dans le territoire anglais.

La légende des enfants-cygnes elle-même est d'origine anglo-saxonne car au moins les premières recherches de Jacob Grimm notent une courte légende du $\mathrm{X}^{\mathrm{e}}$ siècle relatant de la barque sans avirons qui porte un enfant (Lecouteux, 1998 : 125). Les recherches de la source de la légende mènent à travers les légendes saxonnes où on trouve le héros Sceaf qui étymologiquement rappelle le nom de Scandinavie et la région de Schleswig (Ibid., 126) et qui serait le fils des descendants du dieu nordique suprême, Odin. Ce héros est largement connu dans les sagas du nord et il semble être d'une très grande antiquité. Les deux récits, Isomberte espagnole et Beatrix française semblent être les remaniements d'Elioxe qui a l'air d'être la plus proche à l'histoire de Dolopathos. Ce qui diffère Elioxe d'autres

\section{Voir supra.}

18 Il y a encore une autre référence à la généalogie mystérieuse de Godefroi de Bouillon. Celle dans La Chanson d'Antioche où il y a les vers (pendant le siège d'Antioche) : "-Sire ne parlés mais, qu'a mal nel tiegne on, / Molt est de grant parage, par Deu qui fist ne mon. / Vos avés bien oï qui il est ne qui non: / Son avie a duis un cisnes a Nimaie el sablon, / Enmi le plain gravier, el plus maistre donjon, / Tot seul en un batel, ainc n'i ot compaignon, / Bien cauciet et vestu d'un paile d'auqueton, / Plus reluisoit ses ciés que pene de paon, / Ainc Dex ne fist un home de si bele façon. / Le cors ot molt plenier, bien resambla baron. / L'emperere el retint par el gueredon / K'il dona moillier en ceste region, / Un soie parent d'un sien cosin Begon, / Tere bone et fegonde et l'onor de Buillon. ", vv. 7450 - 64. (Duparc-Quioc, 1976 : 372). 
versions, c'est le manque presque total de l'élément chrétien, p.ex. le fait qu'Elioxe est une fée et d'autres éléments qui semblent provenir d'un monde païen et qui n'existent pas dans les versions ultérieures de Beatrix et d'Isomberte. Pourtant, les deux versions de Beatrix, anglaise et française, sont imprégnés avec de l'imaginaire chrétien qui essaie vaguement de remplacer le mystérieux païen. Dans les deux versions on trouve par exemple la biche qui miraculeusement aide l'ermite à sauver les enfants et qui est envoyée par le Christ ou par Dieu. La situation dans la version anglaise quand l'ermite remarque les enfants : "And cryde ofte opon cryste for somme sokour hym to sende, / Il any lyfe were hem lente in Dis worlde lengur. / Thenne an hynde kome fro De woode rennynge fulle swyfte, / And felle be-fore hem adowne Dey drow3e to Pe pappes; " (vv. 111-14) ${ }^{19}$, tandis que dans la française le trouvère décrit le même moment (après la prière d'ermite au Dieu) : «Et quant li sains hermites ot sa proiere faite, / Este vous une cirge ki les enfans alaite / Que Dey i envoia [...]» (vv. $368-70)^{20}$.

Claude Lecouteux qui a fait une recherche historique-littéraire sur le mythe de la fée Melusine, a trouvé des faits importants dans la légende du Chevalier au Cygne où il exclut complètement les sources allemandes, car elles sont trop tardives et elles ne présentent pas, selon la théorie d'épopée de Labarthe, l'épique primaire où la tradition orale est très transparente, mais secondaire, car les récits allemands sont clairement l'œuvre des auteurs et ne font pas partie de la tradition orale (Lecoutoux 1998, 108) ${ }^{21}$. Lecouteux sort du fait que l'élément d'interdiction qui est présent dans la légende de la fée Melusine provient du monde celte et que l'interdiction dans le Chevalier au cygne provient du même modèle. L'interdiction a, selon lui, une marque de finité, du tabou, "une sorte de malédiction, un geis » ${ }^{22}$, qui est présent déjà dans le récit de Beatrix parce que le frère ne va jamais reprendre sa forme humaine et cette " punition » cache en elle un mystère païen qui a sa propre loi fabuleuse. La douleur du cygne qui restera dans sa forme animale est terrible: "But on was alwaye a swanne for fosse of his cheyne. / Hit was doole fot to se De sorow Pat he made; / He bote hym self with his bylle Dat alle his breste blede, " (vv. 358 $60)^{23}$ et dans la version française "Li.I. [une chaîne] $i$ a fali et cil seus est en paine; / Par.III. fois est pasmés, puis brait a grant alaine, / A son bec se demaine, toute la cars li saine. » (vv. $1852-53)^{24}$. Le monde d'au-delà dans lequel se font ces restrictions restera fermé pour les hommes du réel, il se fait une substitution pour l'inconscient et essaie de se débarrasser de ce qu'on pourrait nommer providentialisme chrétien,

19 H. Gibbs : Chevalere Assigne, 1868, 6.

20 Nelson J. A., Mickel E.J., 1977, 139.

21 À comparer : Labarthe, $2005: 16$.

22 Le terme de " geis » désignant un tabou. (Lecoutoux, $1998: 121$ ).

23 Gibbs, $1868: 18$.

24 Nelson et Mickel, $1977: 171$. 
soit il restera, comme le note Georges Dumézil pour le terme d'épopée «en communication constante, dans les deux sens, avec les contes» (Dumézil, 2007 : 19). Dans Beatrix française autant qu'en anglaise on remarque le nom du chevalier Elias qui va mystérieusement perdre son prénom en entrant dans l'histoire française du Chevalier au cygne, mais il le regagne quand les continuateurs français (dans ce cas les jongleurs et les trouvères) inventent la chanson intitulée La Fin d'Elias où le protagoniste rentre dans le monde d'au-delà. Le héros porte le nom d'Enyas dans la version anglaise de Beatrix : "Go brynge hym to his fader courte E loke Dat may be cristened; / And kalle hym Enyas to name for aw3te Pat may be-falle " (vv. 204-4). ${ }^{25}$ La différence de la lettre n'est pas expliquée, ni pourtant la disparition du nom dans la version française. Cependant, les récits de Beatrix, version française et anglaise se ressemblent beaucoup et ils excluent la parenté de Perceval, ce qui est l'élément clé dans les versions allemandes.

Cette mère qui a perdu sa caractéristique féerique dans les versions Beatrix, représente l'élément clé pour découvrir l'inspiration du conte des enfants-cygnes. Il faut souligner aussi que contrairement à la nature très militaire du Cycle de la croisade, cette branche semble très féminine, ayant le protagoniste féminin et on connaît des métamorphoses femmes-cygnes en France médiévale, autant qu'en Irlande. ${ }^{26}$ De même, cet élément d'interdiction provient du monde celtique ce qui a prouve Lecouteux dans l'explication du mythe de la fée Melusine. Mais l'une des sources les plus évidentes qui proviennent du monde celtique, sont des chaînes d'or ou d'argent qui sont innés aux enfants (surtout dans le récit d'Elioxe; et dans le Cycle de la Táin, où il y a les oiseux reliés par les chaînes dorées).

L'autre élément qui révèle des mythologies irlandaises est celui d'un petit navire sans avirons et Ferdinand Lot a découvert un récit irlandais datant du XVIII ${ }^{\mathrm{e}}$ siècle qui raconte l'histoire du roi Lir où on trouve les métamorphoses des enfants-cygnes, la marâtre démoniaque, les enchantements druidiques, les premiers saints chrétiens et à la fin de l'histoire les cygnes changent aux vieillards qui meurent (Lecouteux 1998 :120). À la base d'une recherche étymologique on découvre que la langue gaélique d'Ecosse donne pour le mot du cygne «eala » et en langue irlandaise «ela» (Matthews, 2002) ce qui pourrait présenter l'étymologie inexpliquée dans le contexte de la matière, pour Elioxe et Elias. Le conte irlandais ressemble - dans une certaine mesure - à un conte japonais, Urashima Taro, ce qui prouve que le matériel fabuleux provient d'inconscient et c'est surtout l'élément d'eau qui le rappelle et qui, dans le monde littéraire, fait penser à l'au-delà. Cette matière a pu entrer en continent en $\mathrm{IX}^{\mathrm{e}}$ siècle lors de l'installation des moines irlandais sur les bords du Rhin. Le poème de Beatrix qu'on trouve en Angleterre

Gibbs, 1868 : 10.

26 P.ex. Dans les lais de Graelent, de Guingamor et de Garin (Lecoutoux, 1998 : 118). 
contient ainsi les éléments celtes et anglo-saxons qui se sont répandus sur le continent, pris une nouvelle forme et rentrés à l'île.

Ce transfert des sources n'a évidemment rien de particulier pour l'époque ; c'est l'importance du mythe et du récit qui prend un tel poids pour être toujours intéressant. Les épopées médiévales peuvent de nos jours être un point repère pour les chercheurs littéraires du genre épique car il y a toujours certains contextes qui doivent être transférés. Si l'on prend en considération le fait que les épopées médiévales étaient orales, les légendes comme celles-ci d'Elioxe et de Beatrix pourraient être transmises par le septième art qui représente aujourd'hui, pareillement aux épopées, « la voix » du peuple.

\section{BIBLIOGRAPHIE}

Barron, William R. J. : Chevalere Assigne and The Naissance du Chevalier au Cygne. Medium Avum, vol. 36, No. 1, 1967, pp. 25-37.

Bender, Karl - Heinz : « De Godefroy à Saladin ». Les épopées romanes, volume III, tome 1/2, fascicule 5. Heidelberg, $1986: 35-83$.

Boudoin, Charles : Le triomphe du héros : étude psychanalytique sur le mythe héros et les grandes épopées. Paris, Plon : 1952.

Chibnall, Marjorie, "Empress Matilda : Queen Consort, Queen Mother and Lady of the English» London : Blackwell Publishers, 1993.

Claudel, Paul : Mémoires improvisés. Paris : Gallimard, 1954.

Demurger, Alain : Les Templiers, une chevalerie chrétienne au Moyen Âge. Paris: Éditions du Seuil, 2008.

Dickès, Jean-Pierre : Sainte Ide de Boulogne, mère de Godefroy de Bouillon. Paris : Éditions de Paris, 2004.

Dumézil, Georges : Mythe et Épopée I. L'idéologie des trois fonctions dans les épopées des peuples indo-européens. Paris : Quatro Gallimard, 2007.

Duparc-Quioc, Suzanne : La Chanson d'Antioche. Paris : Librairie orientaliste Paul Geuthner, 1976.

Emplaincourt Edmond A. : The Old French Crusade Cycle. Vol. 9: La Geste du Chevalier au Cygne. Tuscaloosa: University of Alabama Press, 1989.

Gaullier-Bougassas Catherine : Le chevalier au Cygne à la fin du Moyen Âge. La tradition épique, du Moyen Âge au XIX'e siècle. Éd. Suard François. Paris: Honoré Champion éditeur, 2005, pp. 115-146.

Gibbs, Henry H. : The Romance of the Cheualere Assigne. London : Early english text society, 1868.

Guizot, François : Histoire des croisades par Guillaume de Tyr: Vol. 2. Paris: Brière librairie, 1824. 
Hippeau, Céléstin : La Chanson du Chevalier au Cygne et de Godefroid de Bouillon: Vol. 1-2. Genève: Slatkine Reprints, 1969.

Hulbert, James R. : The «West Midland » of the romances. Modern Philology, Vol. 19, No. 1, 1921, pp. 1-16.

Zink, Michel, éd. : La chanson de Roland. Paris: Le livre de poche, 1990.

Labarthe, Judith : L'épopée. Paris : Armand Colin, 2006.

Lecouteux, Claude : Mélusine et le Chevalier au cygne. Paris : Imago, 1998.

Madelénat, Daniel : L'épopée. Paris : Presses universitaires de la France, 1986.

Matthews, John : Celtic totem animals. Boston: Red Wheel Press, 2002.

Mickel, Emanuel J. Jr. et Nelson Jan A. : The Old French Crusade Cycle, Vol. 1, La Naissance du Chevalier au Cygne. Alabama : The University of Alabama Press, 1977.

Mickel, Emmanuel J. Jr. : The Old French Crusade Cycle. Vol.3: Les Enfances Godefroi and Le Retour de Cournumarant. Tuscaloosa: University of Alabama Press, 1999.

Moorman, Charles : The English Alliterative Revival and the Literature of Defeat. The Chaucer Review, Vol. 16, No. 1, 1981, pp. 85-100.

Propp, Vladimir : La Morphologie du conte. Paris : Seuil, 1970.

Tadeja Dermastja

Ljubljana tadeja.dermastja@gmail.com

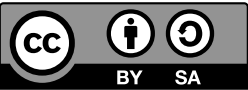

\section{Beatrix, viteški roman v srčiki cikla o križarskih vojnah}

$V$ članku so obravnavani nekateri elementi fantastičnega $v$ srednjeveški veji pisanja o Beatrix, ki je bila integrirana $v$ francoski epski cikel o križarskih vojnah in avtorica jo primerja $\mathrm{z}$ angleško srednjeveško pesmijo $\mathrm{z}$ enakim naslovom. Postavlja si vprašanje zakaj je bila tema te junaške epske pesnitve zanimiva v obeh literarnih okoljih in katere so zgodovinske in književne okoliščine, ki so vplivale na njeno prepoznavnost $\mathrm{v}$ različnih evropskih sredinah.

Ključne besede: chanson de geste, Godefroi de Bouillon, cikel o križarskih vojnah, srednjeveška književnost, Beatrix 